\title{
Temperature-Induced, Selective Assembly of Supramolecular Colloids in Water
}

\author{
Bas G. P. van Ravensteijn, ${ }^{*},, \perp \odot$ Neus Vilanova, ${ }^{\ddagger} \S$ Isja de Feijter, ${ }^{\ddagger}{ }^{\S}$ Willem K. Kegel, ${ }^{\dagger}$
} and Ilja K. Voets $*,+, \S, \|$

${ }^{\dagger}$ Van 't Hoff Laboratory for Physical and Colloid Chemistry, Debye Institute for NanoMaterials Science, Utrecht University, Padualaan 8, $3584 \mathrm{CH}$ Utrecht, The Netherlands

${ }^{\ddagger}$ Institute for Complex Molecular Systems, ${ }^{\S}$ Laboratory of Macromolecular Organic Chemistry, and "Laboratory of Physical Chemistry, Eindhoven University of Technology, P.O. Box 513, 5600 MB Eindhoven, The Netherlands

\section{Supporting Information}

ABSTRACT: In this article, we report the synthesis and physical characterization of colloidal polystyrene particles that carry water-soluble supramolecular $N, N^{\prime}, N^{\prime \prime}$,-trialkyl-benzene1,3,5-tricarboxamides (BTAs) on their surface. These molecules are known to assemble into one-dimensional supramolecular polymers via noncovalent interactions. By tethering the BTAs to charge-stabilized particles, the clustering behavior of the resulting colloids was dictated by a balance between interparticle electrostatic repulsion and the BTA-mediated attractions. Through careful tuning of the dispersing medium's ionic strength, a regime was found in which particle aggregation could be reversibly induced upon heating the dispersion. These findings clearly indicate that hydrophobic interactions, which

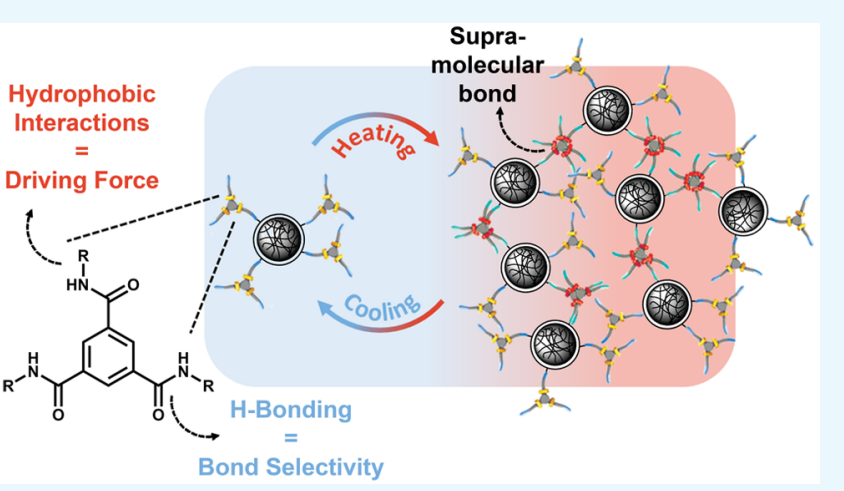
become stronger upon heating, play an important role during the clustering process. Besides the thermoreversible nature of the generated hydrophobic interparticle attractions, we found the clustering to be selective, that is, the BTA-functionalized colloids do not interact with nonfunctionalized hydrophobic polystyrene particles. This selectivity in the association process can be rationalized by the preferred stacking of the surface-tethered BTAs. These selective intermolecular/particle bonds are likely stabilized by the formation of hydrogen bonds, as previously observed for analogous molecular BTA assemblies. The resulting driving force responsible for particle clustering is therefore dual in nature and depends on both hydrophobic attractions and hydrogen bonding.

\section{INTRODUCTION}

Self-assembly of colloidal building blocks into well-defined, predesigned superstructures with tailored functionality is a powerful concept for the preparation of complex materials. ${ }^{1-3}$ By inverse engineering of the desired structures, generally applicable design criteria for the individual building blocks may be formulated. Evidently, one of the difficulties of this bottomup approach lies in the synthesis of the individual building blocks because colloids with elaborate geometries and/or directional interaction potentials are generally required. ${ }^{1-3}$ The demand for sophisticated building blocks sparked the field of synthetic colloid chemistry to develop new systems with tunable (directional) interactions in terms of their strength and range. This already yielded refined colloids that rely on sitespecific DNA hybridization, ${ }^{4}$ magnetic patches, ${ }^{5}$ directional depletion forces, ${ }^{6,7}$ and partial steric stabilization. ${ }^{8}$

Recently, de Feijter et al. expanded the available chemistries to tune interparticle interactions by making use of surfacetethered supramolecular motifs. ${ }^{9}$ Controlling intercolloidal interactions by exploiting supramolecular entities holds potential as a powerful route toward the fabrication of dynamic and responsive mesoscopic materials. Interactions between supramolecular entities are inherently dynamic because they involve noncovalent forces, such as $\pi-\pi$ stacking, hydrophobic interactions, electrostatics, and hydrogen bonding. ${ }^{10}$ To exploit these dynamic features in the colloidal domain, de Feijter et al. immobilized $N, N^{\prime}, N^{\prime \prime}$-trialkyl-benzene-1,3,5-tricarboxamide (BTA) derivatives on hydrophobized silica particles, which were subsequently dispersed in organic solvents. The surfacetethered molecules form interparticle hydrogen bonds making the particles attractive at low temperatures. The aggregation process was thermoresponsive because the formed hydrogen bonds were easily and reversibly broken by heating the particle dispersion. ${ }^{9}$

In this article, we expand the scope of molecular control over colloidal assembly to aqueous systems. Using water as a continuous phase opens the way for exploiting an additional class of noncovalent forces, namely, hydrophobic interactions.

Received: January 30, 2017

Accepted: April 5, 2017

Published: April 28, 2017 
Scheme 1. Schematic Representation of the Hypothesized Aggregation Processes Accessible with the BTA-Functionalized Colloidal Particles ${ }^{a}$

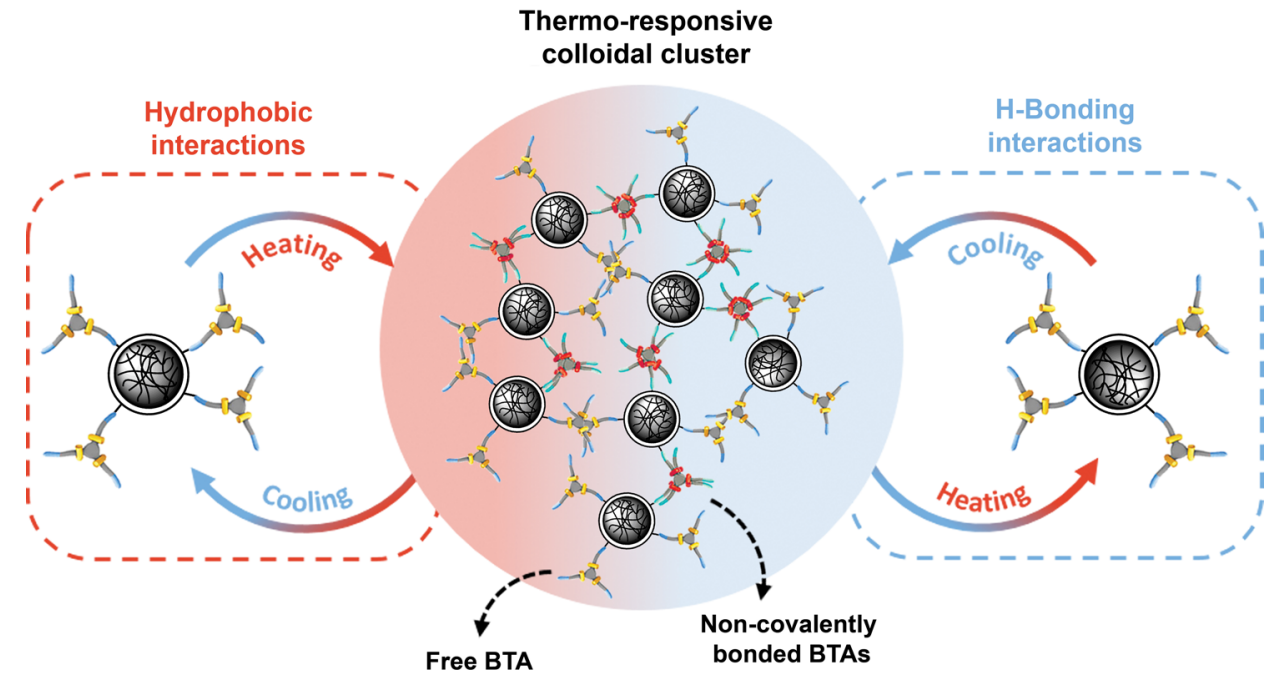

${ }^{a}$ Attraction between colloids that carry water-soluble supramolecular entities on their surface can either be dominated by hydrogen bonds between the amide moieties present in the BTA molecules (blue box; right side of scheme) or by a hydrophobic contribution originating from the dodecyl spacer/aromatic center of the BTA molecules and the temperature-dependent solvation of the oligo(ethylene glycol) fragments (red box; left side of scheme). The strength of both hydrogen-bonding driven and hydrophobically dominated attractions depends on temperature. However, the trends in temperature dependence are reversed. Hydrogen bonds are disrupted at elevated temperatures, whereas hydrophobic attractions generally become stronger upon increasing temperature. Vice versa, at low temperatures, hydrogen bonds should provide stronger attractions between the particles, whereas the hydrophobic contributions are expected to be (significantly) less important.

Although perfected in nature and crucial for the correct folding of proteins ${ }^{11-13}$ and formation of virus capsids, ${ }^{14,15}$ controlling self-assembly in synthetic systems mediated by hydrophobic forces remains a challenge. A limited fundamental insight into hydrophobic forces and the accompanied lack of design criteria for (responsive) hydrophobic materials and molecules are underlying this challenge.

The supramolecular polymerization of water-soluble BTA derivatives was recently studied extensively. ${ }^{16-18}$ These watersoluble derivatives consist of a central benzylic domain, which carries three carboxamide substituents, having hydrophilic oligo(ethylene glycol) and hydrophobic alkyl domains. The amide functionalities are capable of forming intermolecular hydrogen bonds because water is partially "shielded" from this active binding site by the alkyl chains. The terminal oligo(ethylene glycol) fragments ensure water-solubility of the entire building block. Both experiments and simulations elucidated an intricate interplay between hydrogen-bonding and hydrophobic forces during the assembly process. It was suggested that supramolecular polymers initially form as a consequence of hydrophobic attractions and are subsequently further stabilized by the formation of intermolecular hydrogen bonds. ${ }^{17-19}$

By tethering water-soluble BTA molecules to colloidal particles, we envisioned a thermoreversible clustering system that relies on a dual driving force generated by a combination of hydrogen-bonding and hydrophobic interactions between the immobilized BTAs. By studying the temperature-dependent colloidal stability of these supramolecular particles, a regime was found in which effective attractions between the colloids were generated upon heating. This observation strongly implies that hydrophobic interactions dominate over hydrogen bonding at high temperatures (Scheme 1). Reversibility of the aggregation process was evident from spontaneous redispersion of the clusters upon cooling. Although it is generally accepted that hydrophobic interactions are temperature-dependent, using this feature to generate responsive colloidal materials is new to the field. Previous implementations of hydrophobic forces to assemble colloidal objects by, for example, exploiting host-guest complexation or addition of a nonsolvent to polymer-stabilized particles, lack any significant variation in the attraction strength as a function of temperature, limiting the practical use of this attractive force to prepare dynamic materials. $^{20-24}$

In addition to the responsiveness of the hydrophobic attraction, we show that the clustering is also selective. Mixing BTA-functionalized colloids with nonfunctional hydrophobic particles yields clusters solely comprised of supramolecular colloids. This selectivity most probably originates from the hydrogen-bonding capability of the surface-tethered BTAs, providing an additional stabilizing contribution to the interparticle bonds. ${ }^{17-19}$

The combined bond reversibility and selectivity of the BTA supramolecular particles provides a significant advancement in using modular hydrophobic forces to control colloidal aggregation, expanding the toolkit for generating engineered interparticle potentials even further.

\section{RESULTS AND DISCUSSION}

2.1. Synthesis of BTA-Functionalized Colloidal Particles. To expand the scope of molecular control over colloidal assembly to aqueous systems and exploit the dual driving forces generated by an interplay between the hydrogen-bonding and hydrophobic interactions between the immobilized BTAs, we hypothesized that the employed particles should fulfill the following requirements: First, the colloidal surfaces should be as clean as possible. The presence of adsorbed surfactants or polymeric stabilizers might interfere with the attractive surfacegrafted BTA entities. Second, the particles should be easily observable with optical microscopy to probe their clustering 
Scheme 2. Schematic Representation of the Synthesis Procedure toward BTA-Functionalized Colloidal Particles ${ }^{a}$
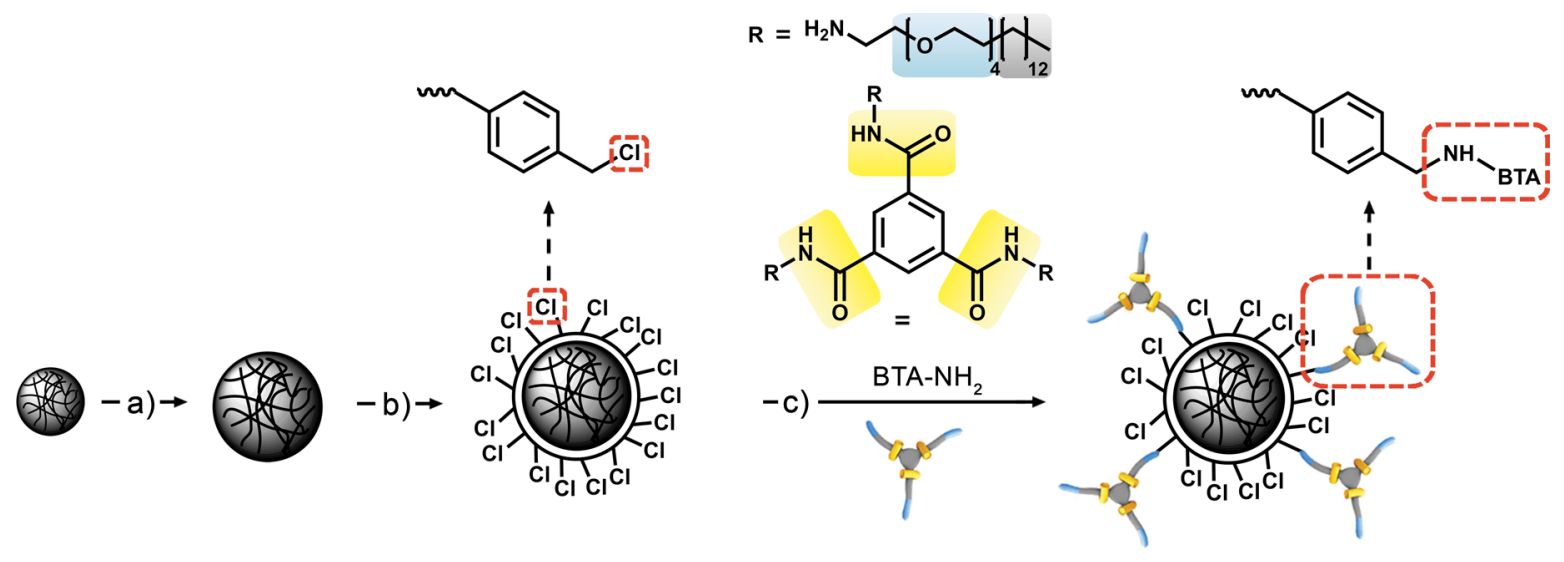

${ }^{a}$ Step (a): seeded emulsion polymerization of styrene (St) and divinylbenzene (DVB) in the presence of cross-linked polystyrene particles (CPs). Step (b): seeded emulsion polymerization of 4-vinylbenzyl chloride (VBC) and DVB in the presence of the previously obtained larger CPs. Step (c): coupling of amino-functionalized BTA- $\mathrm{NH}_{2}$ molecules to the surface of the chlorinated particles via a nucleophilic substitution reaction. The employed BTAs consist of a central benzylic domain, which carries three carboxamide substituents (yellow), having hydrophilic oligo(ethylene glycol) (blue) and hydrophobic dodecyl (gray) segments. All carboxamide arms are functionalized with terminal amines, which are exploited for the coupling reactions with the colloidal surface. The chemical surface entities for each type of colloid are depicted in the top row of the scheme.

behavior in situ. Finally, the particles need to contain a high density of functional groups that can be exploited to tether the BTA supramolecular motifs to the particles.

Large colloidal particles $(1-5 \mu \mathrm{m})$ are typically prepared by conventional dispersion polymerizations, which rely on adsorbed (polymeric) stabilizers to safeguard colloidal stability. ${ }^{25}$ Although easy to observe, the presence of the stabilizers most likely interferes with stacking of the BTA motifs. Dispersions of purely charge-stabilized particles are cleaner in this respect and hence an attractive alternative. These particles are stable due to the presence of charges located directly on the surface of the colloids. Attaching the BTA moieties to these charged surfaces ensures that the molecules protrude from the surface and are free to interact if the colloids can approach closely. The distance upto which colloidal particles can approach each other is easily modulated by electrolyte addition. ${ }^{26}$ Therefore, we speculate that at sufficiently high ionic strengths the envisioned supramolecular charged colloids should become attractive via mutual interaction between the surface-tethered BTA molecules (see next section).

Routinely, charge-stabilized particles are prepared by employing emulsion polymerization-based techniques. ${ }^{25} \mathrm{~A}$ minor disadvantage of emulsion polymerizations is the practical limit to particles with radii of roughly $250 \mathrm{~nm}$. Pushing experimental conditions to exceed this maximum size generally results in unstable and/or polydisperse colloids. To overcome this size limitation and enhance the observability of our colloidal particles with optical microscopy, we employed a seeded emulsion polymerization procedure (Scheme 2, step a). This strategy relies on the step-wise growth of monodispersed crosslinked polystyrene particles (CPs). Starting from CPs with a radius of $225 \mathrm{~nm}$ and employing two subsequent growth steps yielded stable and monodispersed particles with a radius of approximately $400 \mathrm{~nm}$. These particles are readily observable with optical microscopy, making these polystyrene particles ideal candidates to proceed to the next step in which chemical functionality was added to the particles (Scheme 2, step b).

By using an analogous seeded emulsion polymerization as used to grow the CPs, a cross-linked chlorinated shell was deposited onto these particles. 4-Vinylbenzyl chloride (VBC) was employed as the shell monomer, yielding colloids with pending surface benzyl chlorides (Scheme 2, step b, top of the figure). These moieties are versatile chemical handles and open the way to a variety of functionalization chemistries. ${ }^{27,28} \mathrm{We}$ chose to couple the BTAs via a simple nucleophilic aliphatic substitution reaction, exploiting the terminal amine functionality present on the carboxamide substituents of the BTA molecules (BTA- $\mathrm{NH}_{2}$, Scheme 2, step c). Because all arms on the BTA molecules carry an amine, it is possible, in principle, that the BTAs are tethered to the colloidal surface with multiple covalent bonds. We expect that this has no major influence on the eventual aggregation behavior. On the basis of the number of BTAs added during the coupling reaction, the surface density of BTA motifs was estimated to be equal to 1 BTA molecule/ $35 \mathrm{~nm}^{2}$.

This relatively high density ensures easy contact of the BTAs upon approach of other supramolecular colloids.

To prove that the BTA moieties were successfully tethered to the particles, infrared (IR) spectroscopy was used as the analytic tool of choice. For this particular experiment, small colloids (sCPs, particle radius $=31 \mathrm{~nm}$ ) were used to enhance the intensity of the BTA-related signals with respect to those of polystyrene originating from the bulk of the particles (for synthetic details, see Section S1). The IR spectrum obtained for sCPs is typical for polystyrene colloids with aliphatic vibrations located between 2900 and $3200 \mathrm{~cm}^{-1}$ and strong aromaticrelated signals at 1450 and $600-700 \mathrm{~cm}^{-1}$ (Figure 1, bottom black spectrum). After employing these particles as seeds in a seeded emulsion polymerization of $\mathrm{VBC}(\mathrm{sCPs}-\mathrm{Cl})$, the IR spectrum showed the appearance of a characteristic $-\mathrm{CH}_{2}-\mathrm{Cl}$ vibration located at $1266 \mathrm{~cm}^{-1}$ (Figure 1, middle red spectrum). Subsequently, BTA- $\mathrm{NH}_{2}$ was coupled to the surface of the chlorinated particles. To maximize the intensity of BTArelated signals, a large molar excess of BTAs compared with the number of chlorine groups was added. After washing the resulting particles repeatedly to remove any free BTA- $\mathrm{NH}_{2}$, IR spectroscopy revealed the appearance of several new signals. Most notable are the additional vibrations observed at approximately 3300 and $1600 \mathrm{~cm}^{-1}$, corresponding to the 


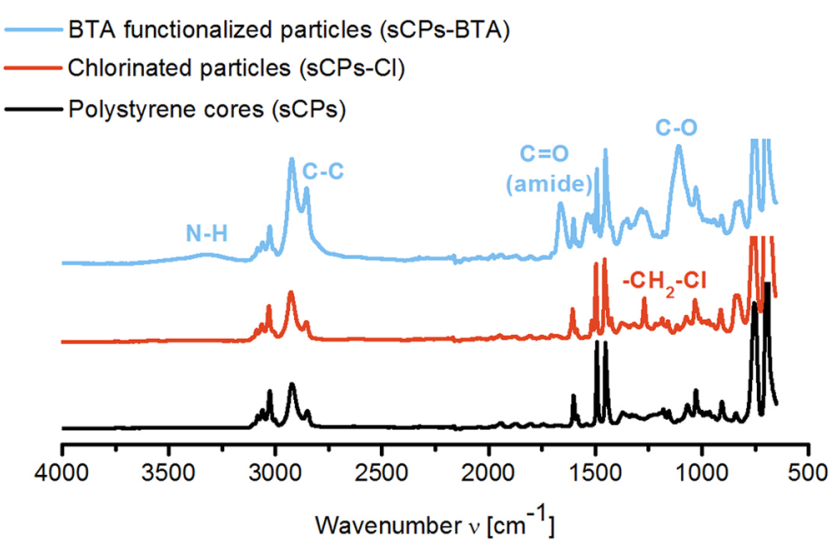

Figure 1. IR spectra of nonfunctionalized polystyrene particles (sCPs, hydrodynamic radius $=31 \mathrm{~nm}$ ) (bottom, black), chlorinated coreshell particles obtained after seeded emulsion polymerization of VBC using $\mathrm{sCPs}$ as seeds (sCPs-Cl, middle, red), and the final BTAfunctionalized colloids after coupling $\mathrm{BTA}-\mathrm{NH}_{2}$ to the chlorinated surface (sCPs-BTA). All characteristic signals are labeled with their corresponding bond/functional group.

amide $\mathrm{N}-\mathrm{H}$ and $\mathrm{C}=\mathrm{O}$ bonds of the attached $\mathrm{BTA}$ entities (Figure 1, top blue spectrum). Furthermore, we observed the appearance of a strong signal located at $1100 \mathrm{~cm}^{-1}$, corresponding to the $\mathrm{C}-\mathrm{O}$ vibrations in the oligo(ethylene glycol) segments of the BTA's pending arms. The dodecyl spacer of these arms is reflected by an increase of the methylene vibration located at $2900 \mathrm{~cm}^{-1}$.

For these small polystyrene particles, IR spectroscopy clearly delivers proof of successful coupling of the BTA entities to the colloidal surface. Because of the analogous synthesis conducted for the larger polystyrene particles, we can safely extrapolate these results to the larger CPs and assume that these colloids are also decorated with BTA moieties.

2.2. Thermoresponsive Behavior of BTA-Functionalized Colloids (CPs-BTA). With the desired BTA-functionalized colloids in hand, we proceeded with investigating their aggregation behavior as a function of temperature and ionic strength. These two experimental variables are the most natural to tune if the effective interaction potential, which acts between the BTA-functionalized colloids, is considered. This effective potential is the sum of an electrostatic repulsion caused by the surface charges on the colloids and an attractive contribution, composed of van der Waals forces and the mutual interaction between the surface-tethered supramolecular entities. From these three interaction terms, the van der Waals forces are hardly tunable. Therefore, we focus on regulating the electrostatics and BTA-mediated interactions to steer the physical behavior of our supramolecular colloidal particles. Naturally, attractions generated by the BTA molecules are only active at small interparticle separations, that is, for a BTAmediated attraction to be generated, a "supramolecular bond" should be formed. Therefore, it is essential that the particles can approach each other as closely as possible. The average minimal distance between individual colloids is determined by the electrostatic repulsion between the particles. The magnitude of the electrostatic repulsion can be controlled by the addition of salt to the dispersion. ${ }^{26}$ Additional ions will screen the surface charges resulting in a diminished interparticle repulsion, enabling closer contact of the colloids. On the basis of this reasoning we hypothesize that there exists an experimental window of salt concentrations where the (aggregation) behavior of the supramolecular colloids is determined by the presence of the BTA molecules on the surface, rather than by other specific colloidal forces.

As explained in the Introduction, similar BTA molecules, as immobilized on the presented colloidal system, form onedimensional aggregates in aqueous environments due to an interplay between hydrogen-bonding and hydrophobic attractions. ${ }^{16,18}$ Both these attractive contributions are temperaturedependent, however, the trend in bonding strength with temperature is generally reversed. Hydrogen bonds form at lower temperatures and vanish if the system is heated. On the contrary, hydrophobic interactions gain in strength with increasing temperature. This trend originates from the fact that interfacial tensions between hydrophobic materials and water $(\gamma(T))$ depend on temperature and can be written as a Taylor expansion around a reference temperature $\left(T_{0}\right)$

$$
\begin{aligned}
\gamma(T) & =\gamma\left(T_{0}\right)+\left(\frac{\partial \gamma}{\partial T}\right)_{T=T_{0}} \times\left(T-T_{0}\right)+\cdots \\
& \approx \gamma\left(T_{0}\right)-\mathrm{s}_{0}\left(T-T_{0}\right)
\end{aligned}
$$

where $s_{0}$ is the surface excess entropy (eq 1$) .{ }^{14}$ This entropy term is negative for most hydrophobic materials, leading to increased surface tensions upon heating. A higher surface tension translates into larger attractions between hydrophobic objects because the energy associated with the formation of a bond between two surfaces scales with the product of the surface tension and the binding area. ${ }^{14}$ The hydrophobic contributions associated with the BTA aggregations originate from the hydrocarbon spacer (highlighted in gray, top of Scheme 2) present in each arm of the BTAs. ${ }^{16,18}$ Additionally, the oligo(ethylene glycol) segments (highlighted in blue, top of Scheme 2) are less hydrated at elevated temperatures. As a consequence, they are less water-soluble and also promote aggregation. ${ }^{29}$

To assay the relative importance of hydrophobic- and hydrogen-bonding contributions to the BTA-mediated interactions between colloids, we performed variable-temperature light scattering experiments on the presented colloidal system, which are extremely sensitive toward clustering. Scattering experiments yield first-order correlation functions $\left(G_{1}\right)$, of which their decay rate depends on the dimensions of the measured objects. $G_{1}$ describes the relation between the average scattering intensity at time $t\left(I_{t}\right)$ and the intensity measured after a certain delay time $\tau\left(I_{t+\tau}\right) \cdot{ }^{30}$ For very short delay times, the correlation is strong $\left(G_{1} \sim 1\right)$, whereas it reduces with increasing delay time until $I_{t}$ and $I_{t+\tau}$ are completely uncorrelated $\left(G_{1}=0\right)$. The obtained correlation functions are effectively a measure of the probability of an object moving a given distance during the period $\tau$. Therefore, the decay rate of a correlation function provides information on the diffusive properties of the particles and hence their hydrodynamic dimensions. ${ }^{30}$ Therefore, a shift of $G_{1}$ toward longer decay times corresponds to slower translational diffusion due to, for example, aggregation of primary particles into clusters.

With this interpretation of the scattering correlation functions in mind, we performed dynamic light scattering (DLS) experiments on highly diluted dispersions of BTAfunctionalized particles in aqueous environments containing 0 , 2.5 , and $10 \mathrm{mM}$ of $\mathrm{NaCl}$ (Figure 2). For each salt concentration, the samples were first measured at $20^{\circ} \mathrm{C}$ and subsequently at $60^{\circ} \mathrm{C}$. As for the molecular studies conducted on these BTA derivatives, $60{ }^{\circ} \mathrm{C}$ was chosen as the upper 
a)

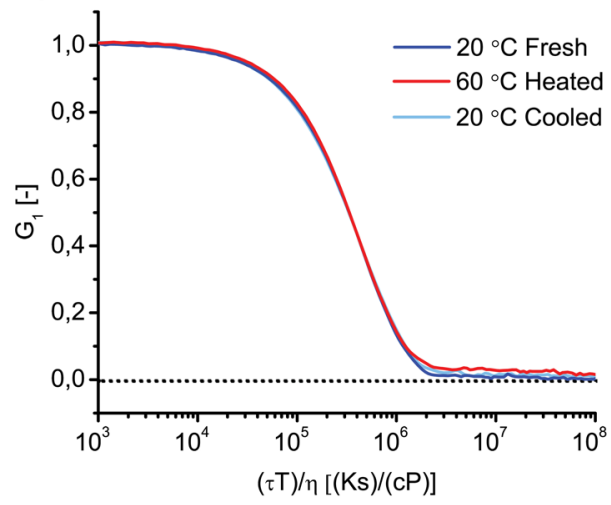

d)

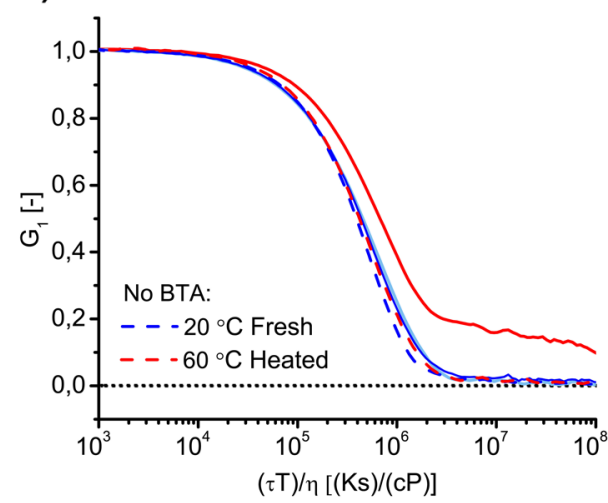

g)

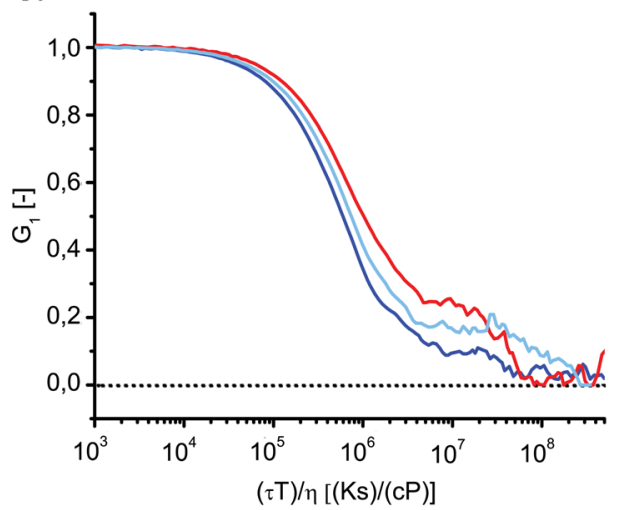

b)

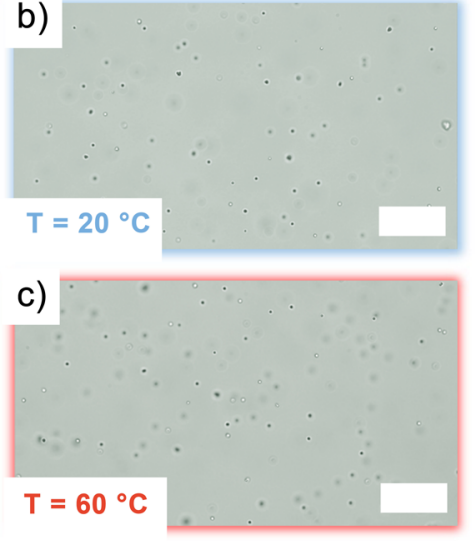

$0 \mathrm{mM}$
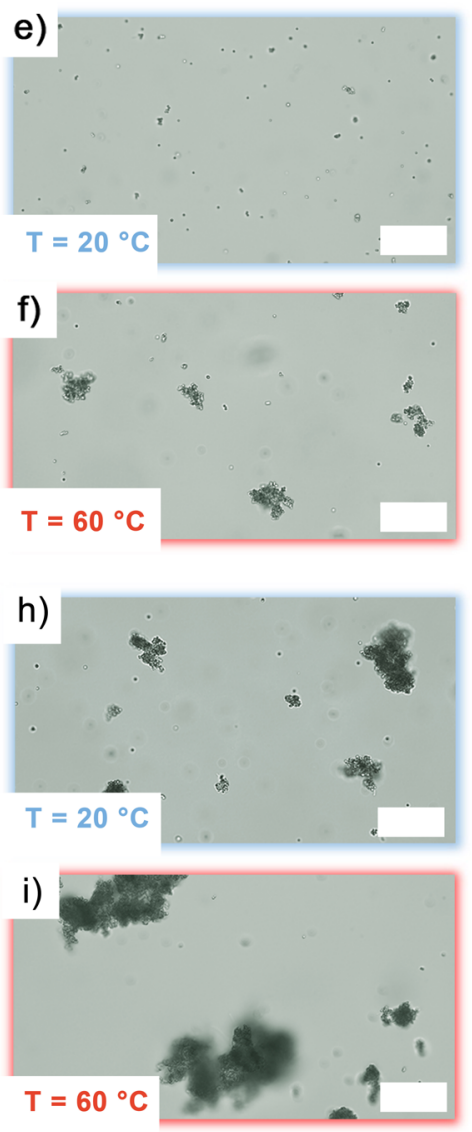

$2.5 \mathrm{mM}$

$10 \mathrm{mM}$

Figure 2. Temperature-dependent first-order correlation functions $\left(G_{1}\right)$ for aqueous dispersions of BTA-functionalized colloidal particles obtained using DLS. The dispersions contained electrolyte $(\mathrm{NaCl})$ concentrations equal to (a) $0 \mathrm{mM}$, (d) $2.5 \mathrm{mM}$, and (g) $10 \mathrm{mM}$. Bright field microscopy images of BTA-functionalized particle dispersions at $20^{\circ} \mathrm{C}$ and subsequent heating to $60{ }^{\circ} \mathrm{C}$. The dispersions contained $\mathrm{NaCl}$ concentrations equal to (b, c) $0 \mathrm{mM}$, (e, f) $2.5 \mathrm{mM}$, and (h, i) $10 \mathrm{mM}$. Scale bar $=10 \mu \mathrm{m}$ for panels b, c, e, f, h, and i.

temperature limit to stay well below the lower critical solution temperature (LCST) of the BTAs $\left(\approx 70{ }^{\circ} \mathrm{C}\right) .{ }^{17,18}$ Heating above the LCST makes the BTAs completely water insoluble, causing uncontrolled hydrophobically mediated aggregation. Furthermore, heating to $60{ }^{\circ} \mathrm{C}$ is sufficient to disrupt (a large fraction of) potentially formed hydrogen bonds. After the heat treatment, the samples were cooled and remeasured at $20^{\circ} \mathrm{C}$ to probe the reversibility of any changes observed in colloidal stability.

In pure water (Figure 2a), smooth, single exponential correlation functions were obtained regardless of the temperature. Measuring a single well-defined characteristic decay time indicates that all colloids effectively diffuse in an equivalent fashion, implying monodispersed and stable particles. As previously mentioned, the lack of aggregation at both low and higher temperatures signifies that the immobilized BTAs cannot interact with each other due to strong electrostatic repulsion between the charged colloids in the absence of any additional salt. ${ }^{26}$ Also, on the microscopic level, temperatureindependent colloidal stability was evident as was concluded from Figure $2 b, c$. These figures show typical optical microscopy images of the dispersion at $20^{\circ} \mathrm{C}$ and after subsequent heating to $60{ }^{\circ} \mathrm{C}$, respectively. 


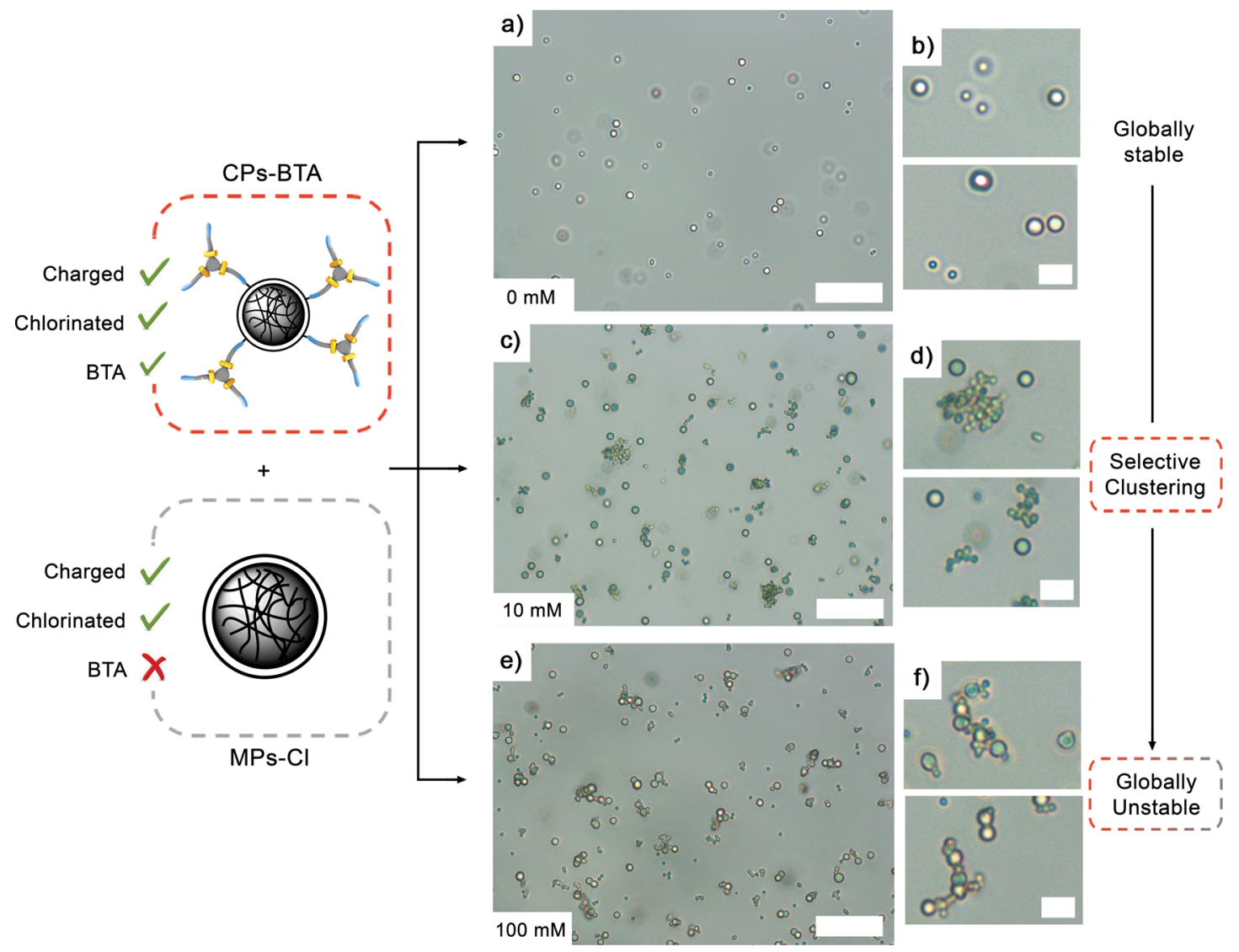

Figure 3. Aggregation behavior of a mixed dispersion containing BTA-functionalized colloids and larger nonfunctionalized polystyrene microspheres as a function of the ionic strength of the dispersing medium. As schematically depicted on the left of the figure, both types of colloids are chlorinated and charged. The only significant difference, apart from their size, is the presence or absence of surface BTA entities. Microscopy images of the mixed dispersion (a) without the addition of salt, (c) in a dispersing medium containing approximately $10 \mathrm{mM}$ and (e) $100 \mathrm{mM} \mathrm{NaCl}$. Panels (b), (d), and (f) contain enlarged images of the dispersions shown in panel (a), (c), and (e), respectively. Scale bar $=10 \mu \mathrm{m}$ for panels (a), (c), and (e) and $4 \mu \mathrm{m}$ for panels (b), (d), and (f).

Increasing the salt concentration to $2.5 \mathrm{mM}$ and measuring at $20{ }^{\circ} \mathrm{C}$ did not significantly alter the shape of the correlation function (Figure 2d, dark blue curve), indicating that the particles were still stable under these conditions. An exemplary light microscopy image shown in Figure 2e confirms this, although the appearance of small clusters suggests that the dispersion is on the edge of colloidal stability. Upon repeating the DLS measurement at $60{ }^{\circ} \mathrm{C}$, a significant tail in the correlation function was observed, implying the formation of aggregates (Figure 2d, red curve). Aggregates are by definition larger than individual particles and therefore diffuse more slowly compared with monomeric colloids. This slower movement translates into a slower decay of the correlation function, as explained before. Because the observed aggregation is purely diffusive, the formed clusters are of random size and geometry, each having their own characteristic decay time. This translates into a noisy tail of the correlogram. The erratic data at long delay times is enhanced even further by the fact that the aggregates grow during data acquisition. Colloidal clustering was also evident from optical microscopy as shown in Figure $2 \mathrm{f}$.

Remeasuring the dispersion after cooling resulted in disappearance of the tail, and the obtained correlogram (Figure $2 \mathrm{~d}$, light blue curve) overlaps with the one previously measured for the fresh dispersion at $20^{\circ} \mathrm{C}$.

These temperature-dependent DLS measurements suggest that mutual attractions are introduced between BTA-function- alized colloids upon raising the temperature. Furthermore, the aggregation process is reversible because redispersion of the colloids occurs if the heated dispersion is cooled. Thus, at a salt concentration equal to $2.5 \mathrm{mM}$, the electrostatic repulsion is apparently just dominant at low temperatures, whereas the increased hydrophobic-mediated attraction generated by heating the particles drives the aggregation at higher temperatures.

The key role of the surface-tethered BTA molecules in the temperature-dependent aggregation process was validated by DLS measurements on the bare chlorinated colloids (Figure 2d, striped curves). The smooth single decaying correlation functions obtained at both temperatures prove that a salt concentration of $2.5 \mathrm{mM}$ is insufficient to overcome the electrostatic repulsion between the chlorinated particles. The attractions generated between the CPs-BTA particles must therefore be mediated by the immobilized BTA molecules. Furthermore, the correlograms measured for the chlorinated particles overlapped perfectly with the curves obtained for the BTA-functionalized colloids at room temperature. This implies that eventhough the BTA molecules are, in principle, able to interact, the supramolecular colloids behave as repulsive, charge-stabilized particles at low temperatures.

Diminishing the electrostatic repulsion even further by increasing the salt concentration to $10 \mathrm{mM}$ yields colloidal aggregates regardless of the temperature (Figure $2 \mathrm{~g}-\mathrm{i}$ ). 

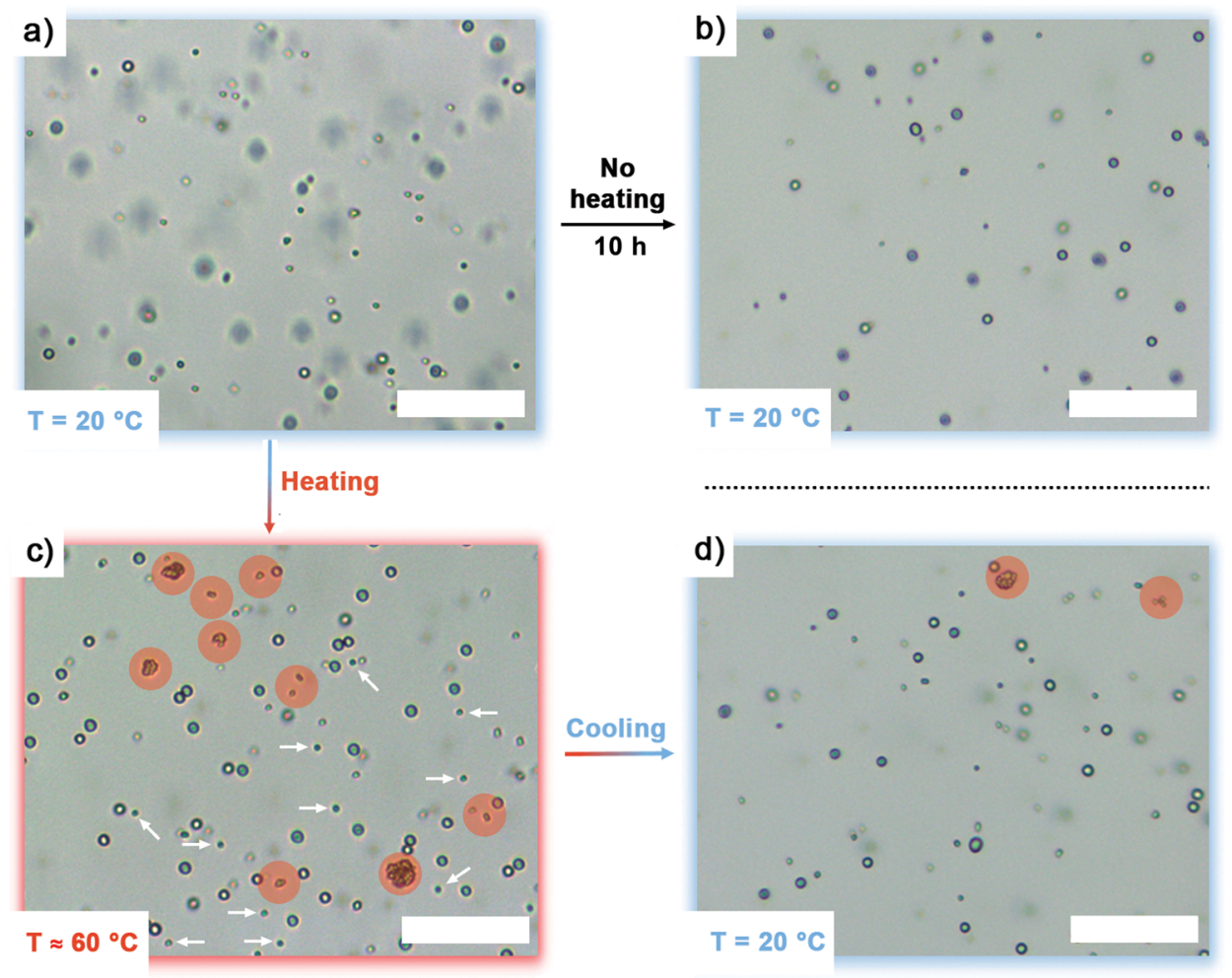

Figure 4. In situ observation of the temperature-dependent colloidal stability of a dispersion containing both BTA-functionalized colloids and larger nonfunctionalized polystyrene microspheres. The dispersion medium contained $2.5 \mathrm{mM}$ of NaCl . (a) Bright field microscopy image of the mixed dispersion at $20{ }^{\circ} \mathrm{C}$. (b) An equivalent sample as shown in panel (a), after equilibration for roughly $10 \mathrm{~h}$ at $20^{\circ} \mathrm{C}$. (c) Microscopy image of the dispersion shown in panel (a), after heating to approximately $60^{\circ} \mathrm{C}$. Monomeric BTA-functionalized particles (CPs-BTA) are highlighted by the white arrows. Clusters of the smaller BTA-functionalized colloids are highlighted with the red circles. (d) Typical appearance of the dispersion obtained after subsequent cooling of the dispersion shown in panel (c). Scale bar $=10 \mu \mathrm{m}$ for all panels.

Evidently, the hydrophobic contributions and possibly hydrogen-bonding interactions are already strong enough to dominate over the screened electrostatic repulsion at room temperature. In agreement with the DLS experiments performed at $2.5 \mathrm{mM}$, aggregation is more pronounced at elevated temperatures and macroscopic flocculates are observed after heating (Figure 2i).

Additional evidence for a modest contribution of hydrogen bonding in the aggregation process was provided by remeasuring a fresh DLS sample containing $10 \mathrm{mM}$ of $\mathrm{NaCl}$ at room temperature in the presence of $100 \mathrm{mM}$ of hexafluorisopropanol (HFIP). HFIP is known for its high efficiency in disrupting hydrogen bonds. ${ }^{31}$ Only if hydrogen bonding would be responsible for the observed aggregation of BTA-covered colloids, the addition of HFIP should result in (partial) recovery of colloidal stability. However, this decreased tendency toward aggregation was not observed (see Section S2, Figure S1), further underlining that the hydrophobic term is the dominant attractive contribution driving colloidal clustering under the described experimental conditions.

2.3. Selective Aggregation of BTA-Functionalized Colloids (CPs-BTA). The results presented in the previous section provided clear experimental evidence that the BTAmediated aggregation is largely driven by hydrophobic attractions. Nevertheless, we expect the generated attractions between the supramolecular colloids to be selective. Recently, experimental and simulation studies on analogous molecular BTA systems revealed the importance of hydrogen bonding in stabilizing the formed supramolecular polymers. ${ }^{19}$ Despite the fact that hydrogen bonds were not an absolute necessity for clustering, the ability of forming intermolecular hydrogen bonds strongly enhances the stability and structural integrity of the formed assembly. Extrapolating these findings to the supramolecular colloids, we hypothesize that the selectivity imprinted by the surface-immobilized BTA motifs translates into a preference to cluster with themselves instead of with other hydrophobic objects.

To verify this hypothesis, we prepared a mixed dispersion containing CPs-BTA colloids and significantly larger, micronsized particles, which were not functionalized with the supramolecular moieties (MPs-Cl). The surfaces of both the CPs-BTA and MPs-Cl particles are chemically equivalent on the basis of their (surface) composition and comparable $\zeta$ potentials, except for one key difference, which is the presence or absence of the BTA moieties. Synthetic details of the procedure followed to prepare $\mathrm{MPs}-\mathrm{Cl}$ and a comparison with the smaller CPs-BTA particles can be found in Section S3.

Mixing particles with similar surface characteristics and therefore physical properties is key to probing any preferential aggregation. The use of particles that can be distinguished from each other on the basis of exclusively their size is also beneficial in this respect because we do not need to tag the particles with additional markers, for example, hydrophobic fluorescent dyes that could influence the interparticle interactions.

The aggregation behavior of particle dispersions containing CPs-BTA and MPs-Cl as a function of the background electrolyte concentration is summarized in Figure 3. Figures $3 \mathrm{a}$ and $\mathrm{b}$ show typical optical microscopy images of the mixed 
dispersion without the addition of any salt. We found a globally stable dispersion without any clustering, as was expected, on the basis of the relatively high $\zeta$ potentials of both types of particles. Both CPs-BTA and MPs-Cl are repulsive toward themselves and the other species.

The situation changes if the ionic strength is raised to approximately $10 \mathrm{mM}$. In agreement with the DLS data presented in Figure 2g, BTA-functionalized particles become mutually attractive and start to form colloidal clusters (Figure $3 \mathrm{c}, \mathrm{d})$. On the contrary, the $\mathrm{MPs}-\mathrm{Cl}$ colloids remain welldispersed and do not participate in the cluster formation. Despite the fact that the attractions between CPs-BTA particles are largely being driven by hydrophobic interactions (see previous section), contacts between hydrophobic bare polystyrene surfaces and BTAs are not generated or at least not stable enough to form mixed aggregates with an observable lifetime. The observed selectivity implies that the clustering of CPs-BTA is driven by mutual interactions between the surfacetethered BTAs.

Upon further increase of the salt concentration to $100 \mathrm{mM}$, the selectivity in cluster formation was lost. At these high ionic strengths, the electrostatic repulsion between all species was diminished to such an extent that all particles became unstable. In this situation mixed aggregates are formed, which consist of both MPs-Cl and CPs-BTA particles (Figure 3e,f).

In the previous section, temperature-induced aggregation was demonstrated for salt concentrations at which electrostatic repulsion only dominates at room temperature. In principle, it should therefore also be possible to induce selective aggregation in a mixed dispersion of CPs-BTA and MPs-Cl by simply increasing the temperature. Conducting an in situ heating experiment, where a thin capillary containing the mixed dispersion was heated while being observed with optical microscopy, revealed that this selective, temperature-induced aggregation was indeed achievable. The salt concentration of the used dispersion was equal to $2.5 \mathrm{mM}$, which was previously identified as the critical salt concentration to induce thermoreversible clustering of CPs-BTA. Figure 4a shows the globally stable mixed dispersion before it was subjected to the heat treatment. Upon heating the capillary, the formation of small clusters was observed, which again consisted of solely the smaller BTA-functionalized particles (Figure 4c, highlighted with the red circles). Upon cooling the dispersion back to room temperature, the majority of the clusters disappeared. Complete reversibility was not observed, probably caused by the fact that the BTA-functionalized particles were on the edge of colloidal instability when dispersed in a continuous phase containing 2.5 $\mathrm{mM}$ of $\mathrm{NaCl}$ (Figure 2f). Nevertheless, this seemingly thermoreversible clustering is in agreement with the DLS measurements of CPs-BTA conducted under similar conditions (Figure 2d).

Storing the mixed dispersion in a capillary without subjecting it to any heat treatment did not induce clustering (Figure $4 b$ ). This corroborates that aggregation is indeed caused by a temperature-controlled hydrophobic interaction between the supramolecular colloidal particles.

\section{CONCLUSIONS}

In this article, we showed the successful synthesis of chargestabilized colloidal particles surface-functionalized with watersoluble supramolecular BTA moieties. On the basis of molecular studies performed on equivalent BTAs, we expected these molecules to provide an attractive force between the particles, driven by a combination of hydrogen-bonding and hydrophobic interactions. By studying the temperature-dependent clustering behavior of the BTA-functionalized particles, the dominant driving force for aggregation at elevated temperatures was identified. Tuning the electrostatic repulsion by varying the salt concentration of the dispersing medium, led to a situation in which the surface-tethered BTA moieties generated an attractive mutual interaction between the particles. However, clustering was only induced upon heating the dispersion. Evidently, this temperature dependence of the colloidal stability strongly points toward hydrophobically mediated attractive forces because these interactions tend to increase in strength upon raising the temperature. Nevertheless, the hydrogenbonding capability of the surface-immobilized BTAs seems important in the formation of colloidal aggregates. We showed that under appropriate conditions the interactions generated between the BTA molecules are not only thermoreversible but also selective. The supramolecular colloids preferentially cluster with identical particles rather than with other non-BTA containing hydrophobic objects. This selectivity in bond formation cannot be explained on the basis of solely hydrophobic interactions and is most probably caused by the formation of stabilizing intermolecular/particle hydrogen bonds between surface-grafted BTAs. In analogy with advanced biological self-assembled systems, for example, virus capsids, this colloidal system relies on a delicate balance between noncovalent forces to steer the assembly process. The combined bond reversibility and selectivity of our supramolecular colloids therefore provides a significant advancement in using modular hydrophobic forces to control and manipulate colloidal aggregation.

Evidently, the novel BTA-mediated interactions are not limited to spherical particles only. By extending the presented results to more elaborate colloidal systems, for example, patchy particles, a synthetic pathway to design colloids that can form directional, hydrophobic-mediated bonds is anticipated. In contrast to the random aggregates formed by spherical particles, these patchy particles potentially form well-defined, thermoreversible supracolloidal superstructures that resemble biological assemblies not only in their interplay between a variety of noncovalent forces but also in their degree of structural ordering.

\section{EXPERIMENTAL SECTION}

4.1. Materials. Styrene (St, 99\%), divinylbenzene (DVB, $55 \%$ mixture of isomers, tech. grade), and 4-vinylbenzyl chloride (VBC, $(\geq 90 \%$, tech. grade) were purchased from Sigma-Aldrich. Sodium dodecylsulfate (SDS) from $\mathrm{BDH}$ was used. Potassium persulfate (KPS, >99\% for analysis), sodium bisulfite $\left(\mathrm{NaHSO}_{3}\right.$, ACS reagent), and dimethyl sulfoxide (DMSO, 99.7\%) were purchased from Acros Organics. 1,1,1,3,3,3-Hexafluoro-2-propanol (HFIP, $\geq 99 \%$, for (peptide) synthesis) from Carl Roth was used. Finally, sodium chloride $(\mathrm{NaCl}$, ACS reagent $\geq 99.5 \%)$ was purchased from Merck. All chemicals were used as received. The water used throughout all syntheses was purified using a Milli-Q water purification system.

4.2. Synthesis of Chlorinated Particles (CPs-Cl). Crosslinked polystyrene (CPs) core particles were synthesized based on of a standard emulsion polymerization method. ${ }^{27,32}$ Slight modifications to the reported procedure were applied to yield CPs colloids with larger dimensions. A $500 \mathrm{~mL}$ round-bottom flask equipped with a magnetic stir bar was placed in an oil bath 
at $80{ }^{\circ} \mathrm{C}$. Water $(200 \mathrm{~mL})$ was charged into the reactor and allowed to reach the bath temperature. Styrene $(23 \mathrm{~mL}, 0.2$ $\mathrm{mol})$, DVB $(0.7 \mathrm{~mL}, 5 \mathrm{mmol})$, and SDS $(0.125 \mathrm{~g}, 0.43 \mathrm{mmol})$ dissolved in water $(50 \mathrm{~mL})$ were added. The mixture was allowed to heat up to the temperature of the bath. Finally, the addition of KPS (0.39 g, $1.4 \mathrm{mmol})$ dissolved in water $(37.5$ $\mathrm{mL}$ ) initiated polymerization. The reaction was allowed to continue for $24 \mathrm{~h}$ at $80^{\circ} \mathrm{C}$. The resulting dispersion had a solid content of $7 \%$ (measured gravimetrically). The resulting particles had a radius of $225 \mathrm{~nm}$ with a polydispersity of $3.8 \%$, as determined with transmission electron microscopy (TEM).

Subsequently, the prepared particles were used as seeds in a seeded emulsion polymerization of styrene and DVB. To this end, crude CPs dispersion $(12.5 \mathrm{~mL}$, solid content $=7 \%)$ and water $(5 \mathrm{~mL})$ were introduced into a $50 \mathrm{~mL}$ round-bottom flask equipped with a magnetic stir bar. The mixture was degassed with nitrogen for $30 \mathrm{~min}$. Styrene $(1.25 \mathrm{~mL}, 0.01$ mol) premixed with DVB $(25 \mu \mathrm{L}, 0.17 \mathrm{mmol})$ was injected under inert atmosphere. The seeds were swollen for $1.5 \mathrm{~h}$ at 30 ${ }^{\circ} \mathrm{C}$, after which the temperature was raised to $60{ }^{\circ} \mathrm{C}$. When this temperature was reached, KPS $(20 \mathrm{mg}, 0.07 \mathrm{mmol})$ and sodium bisulfite $(15 \mathrm{mg}, 0.14 \mathrm{mmol})$ dissolved in water $(1.25 \mathrm{~mL})$ were added. The reaction was allowed to run for $12 \mathrm{~h}$. The resulting particles were washed by centrifugation and redispersion in water. The solid content of the dispersion was adjusted to 5\%. Particles with a hydrodynamic radius of $340 \mathrm{~nm}$ and polydispersity index (PDI) of 0.09 were obtained as measured with DLS. The described seeded emulsion polymerization was repeated once more using the dispersion obtained after the first seeded polymerization $(12.5 \mathrm{~mL}$, solid content $=5 \%)$. In this second growth step, added quantities and reaction times were equal to those described for the first growth step. Growing the polystyrene particles for the second time yielded colloids with a hydrodynamic radius of $395 \mathrm{~nm}$ and PDI of 0.1.

In the final reaction step, benzyl chloride moieties were introduced at the surface of the synthesized particles. ${ }^{25}$ The washed dispersion obtained after the second seeded emulsion polymerization $(10 \mathrm{~mL}$, solid content $=5 \%)$ and water $(4 \mathrm{~mL})$ were introduced into a $25 \mathrm{~mL}$ round-bottom flask equipped with a magnetic stir bar. The mixture was degassed with nitrogen for $30 \mathrm{~min}$. VBC $(0.4 \mathrm{~mL}, 2.8 \mathrm{mmol})$ premixed with DVB ( $8 \mu \mathrm{L}, 0.06 \mathrm{mmol})$ was injected under inert atmosphere. The seeds were swollen for $1 \mathrm{~h}$ at $30{ }^{\circ} \mathrm{C}$, after which the temperature was raised to $60{ }^{\circ} \mathrm{C}$. When this temperature was reached, KPS (16 mg, $0.06 \mathrm{mmol})$ and sodium bisulfite $(12 \mathrm{mg}$, $0.11 \mathrm{mmol})$ dissolved in water $(1 \mathrm{~mL})$ were added. The reaction was allowed to run for $4 \mathrm{~h}$. The resulting particles were washed by centrifugation and redispersion in water. The solid content of the dispersion was adjusted to 5\%. A hydrodynamic particle radius of $470 \mathrm{~nm}$ and PDI of 0.08 were measured using DLS. The relatively large size enabled visualization of the colloids with optical microscopy. Stable dispersions, without any signs of aggregation were obtained. Furthermore, the presence of the chlorine groups was confirmed using Fourier transform infrared (FT-IR) spectroscopy $\left(1266 \mathrm{~cm}^{-1}\right)$.

4.3. Synthesis of BTA-NH $\mathrm{NH}_{2}$. N,N',N" $N^{\prime \prime}$ Tris(1-amino3,6,9,12-tetraoxatetracosan-24-yl) benzene-1,3,5-tricarboxamide (BTA- $\mathrm{NH}_{2}$, chemical structure see Scheme 2) was synthesized, as previously reported by Albertazzi et al. ${ }^{33}$ and Baker et al. ${ }^{16}$ The molecule comprises a benzylic group with three carboxamide substituents linked to an alkyl spacer of 12 carbons, which in turn is linked to a hydrophilic segment consisting of 4 oligo(ethylene glycol) groups. These hydrophilic segments are end-capped with primary amines.

4.4. Functionalization of $\mathrm{CPs}-\mathrm{Cl}$ with $\mathrm{BTA}-\mathrm{NH}_{2}$ (CPsBTA). A dispersion containing CPs-Cl particles $(0.5 \mathrm{~mL}$, solid content $=5 \%)$ was transferred from water to DMSO $(0.4 \mathrm{~mL})$ by centrifugation and redispersion. The DMSO dispersion was introduced into a Schlenk flask and degassed by evacuation and refilled with nitrogen multiple times. In a separate Schlenk flask, a stock solution of BTA-NH $\mathrm{NH}_{2}(1 \mathrm{mg}, 0.8 \mu \mathrm{mol})$ in DMSO (10 $\mathrm{mL}$ ) was introduced and subsequently degassed by evacuation and refilled with nitrogen multiple times. To start the coupling reaction between the surface benzyl chloride moieties and the pending amine functionalities of the BTA molecules, BTA stock solution $(0.1 \mathrm{~mL})$ was injected into the DMSO CPs-Cl dispersion under inert atmosphere. The obtained reaction mixture was stirred for $24 \mathrm{~h}$ at $70{ }^{\circ} \mathrm{C}$. After this period, the particles were washed with ethanol and water. Introduction of the BTA moieties onto the particle surface did not influence the colloidal stability or hydrodynamic dimensions of the particles compared to those of the bare chlorinated $\mathrm{CPs}-\mathrm{Cl}$ particles as determined with DLS.

4.5. Characterization. IR spectra were obtained using a PerkinElmer Frontier FT-IR/FIR spectrometer operating in the attenuated total reflectance (ATR) mode. All measurements were performed on powders obtained by drying the corresponding colloidal dispersion.

TEM pictures were taken with a Philips Technai10 electron microscope typically operating at $100 \mathrm{kV}$. Bright field images were recorded using a SIS Megaview II CCD camera. The samples were prepared by drying a drop of diluted aqueous particle dispersion on top of Formvar (R1202 Agar Scientific)coated copper grids (square 300 mesh, G2300C Agar Scientific).

DLS was performed using a Malvern Zetasizer Nano equipped with a $633 \mathrm{~nm}$ wavelength laser. Highly diluted aqueous dispersions were measured in glass cuvettes at temperatures of 20 and $60{ }^{\circ} \mathrm{C}$ in the backscattering mode $\left(173^{\circ}\right)$. An equilibration time of $5 \mathrm{~min}$ was applied before starting each experiment to ensure that the dispersions were at the set temperature. DLS measurements included 15 acquisitions conducted 7 times to determine the hydrodynamic radius of the colloids throughout the described synthesis procedure. The correlation functions were averaged and analyzed according to the method of cumulants to obtain a harmonic $Z$-average radius and the corresponding PDI. ${ }^{34}$

For the DLS experiments conducted to probe the thermoreversible aggregation of BTA-functionalized colloids, the number of runs was adjusted according to the aggregation or stabilization kinetics of the sample. However, physically meaningful values for the harmonic $Z$-average diameter and PDI could not be obtained for aggregated dispersions, which is why we reported the first-order correlation functions for these experiments instead. The autocorrelation functions $\left(G_{1}(\tau)\right)$ decay from 1 at short delay times $(\tau)$ to 0 at long delay times. To facilitate a comparison between the individual DLS experiments performed at different temperatures, $G_{1}(\tau)$ is plotted as a function of the reduced delay time $t^{\prime}$, which is the delay time $\tau$ multiplied by the temperature $(T)$ and divided by the viscosity $(\eta)$ of the dispersing medium (eq 2). ${ }^{35}$

$$
t^{\prime}=\frac{\tau T}{\eta}
$$


Electrophoretic mobility measurements were conducted on the same Malvern Zetasizer Nano instrument as utilized for DLS. Measurements were performed in disposable folded capillary cells at $20{ }^{\circ} \mathrm{C}$. Seven runs of at least 50 individual measurements were conducted to obtain a statistically reliable zeta $(\zeta)$ potential. Electrophoretic mobilities were measured in Milli-Q water. Because the product of the particle radius and the inverse Debye screening length $(\kappa R)>1$ for the measured samples, the Smoluchowski limit of the Henry equation was used to convert the measured electrophoretic mobilities to $\zeta$ potentials. $^{36}$

To probe the effect of heating on the BTA-functionalized colloids in situ, a diluted particle dispersion was introduced into a capillary $\left(0.05 \times 1.00 \mathrm{~mm}^{2}(\mathrm{CM}\right.$ Scientific $\left.)\right)$, which was subsequently sealed with chemical resistant two-component glue. The capillary was mounted on the heating element of a Linkam THMS 600 heating stage, which was placed on the table of a Nikon Eclipse Ti-U inverted optical microscope equipped with an InfinitiX scout camera. The heating stage was connected to a Linkam TP 93 controller, which enabled to heat and cool the capillary while monitoring the sample. A 40X objective with a relatively long working distance $(2.7-3.7 \mathrm{~mm}$ ) was used to prevent heating up of the objective during these experiments.

For the optical microscopy images shown throughout the article, which did not require in situ observation of colloidal aggregation, samples were prepared by placing a drop of diluted dispersion on a microscopy glass. A $40 \times$ or $60 \times$ magnification objective was used.

\section{ASSOCIATED CONTENT}

\section{S Supporting Information}

The Supporting Information is available free of charge on the ACS Publications website at DOI: 10.1021/acsomega.7b00111.

Synthetic procedures to prepare significantly smaller chlorinated polystyrene colloids and their functionalization with BTA-NH $\mathrm{N}_{2}$, the effect of HFIP on the stability of the formed supramolecular clusters as probed with DLS and optical microscopy and the synthesis of micronsized, chlorinated polystyrene colloids (MPs-Cl) (PDF)

\section{AUTHOR INFORMATION}

\section{Corresponding Authors}

*E-mail: bas.van.ravensteijn@gmail.com (B.G.P.v.R.).

*E-mail: i.voets@tue.nl (I.K.V.).

ORCID ${ }^{\circ}$

Bas G. P. van Ravensteijn: 0000-0001-9024-3927

\section{Present Address}

${ }^{\perp}$ Department of Chemical Engineering, University of California at Santa Barbara, Santa Barbara, California 93105, United States (B.G.P.v.R.).

\section{Author Contributions}

The manuscript was written through contributions of all authors. All authors have given approval to the final version of the manuscript.

\section{Funding}

This work was financially supported by The Netherlands Organization for Scientific Research (NWO ECHO-STP Grant 717.013.005, NWO VIDI Grant 723.014.006).

\section{Notes}

The authors declare no competing financial interest.

\section{ACKNOWLEDGMENTS}

The authors thank Fuqiang Chang for synthesizing the micronsized, charge-stabilized chlorinated particles ( $\mathrm{MPs}-\mathrm{Cl}$ ) and Laura Neumann for the synthesis of the BTA derivative (BTA$\mathrm{NH}_{2}$ ).

\section{REFERENCES}

(1) Glotzer, S. C.; Solomon, M. J. Anisotropy of building blocks and their assembly into complex structures. Nat. Mater. 2007, 6, 557-562.

(2) Yi, G.-R.; Pine, D. J.; Sacanna, S. J. Recent progress on patchy colloids and their self-assembly. J. Phys.: Condens. Matter 2013, 25, No. 193101.

(3) Pawar, A. B.; Kretzschmar, I. Fabrication, assembly, and application of patchy particles. Macromol. Rapid Commun. 2010, 31, $150-168$.

(4) Wang, Y.; Wang, Y.; Breed, D. R.; Manoharan, V. N.; Feng, L.; Hollingsworth, A. D.; Weck, M.; Pine, D. J. Colloids with valence and specific directional bonding. Nature 2012, 491, 51-56.

(5) Sacanna, S.; Rossi, L.; Pine, D. J. Magnetic click colloidal assembly. J. Am. Chem. Soc. 2012, 134, 6112-6115.

(6) Kraft, D. J.; Ni, R.; Smallenburg, F.; Hermes, M.; Yoon, K.; Weitz, D. A.; van Blaaderen, A.; Groenewold, J.; Dijkstra, M.; Kegel, W. K. Surface roughness directed self-assembly of patchy particles into colloidal micelles. Proc. Natl. Acad. Sci. U.S.A. 2012, 109, 1078710792.

(7) Wolters, J. R.; Avvisati, G.; Hagemans, F.; Vissers, T.; Kraft, D. J.; Dijkstra, M.; Kegel, W. K. Self-assembly of "Mickey Mouse" shaped colloids into tube-like structures: Experiments and simulations. Soft Matter 2015, 11, 1067-1077.

(8) van Ravensteijn, B. G. P.; Kegel, W. K. Versatile procedure for site-specific grafting of polymer brushes on patchy particles via atom transfer radical polymerization (ATRP). Polym. Chem. 2016, 7, 28582869.

(9) de Feijter, I.; Albertazzi, L.; Palmans, A. R. A.; Voets, I. K. Stimuli-responsive colloidal assembly driven by surface-grafted supramolecular moieties. Langmuir 2015, 31, 57-64.

(10) Brunsveld, L.; Folmer, B. J. B.; Meijer, E. W.; Sijbesma, R. P. Supramolecular polymers. Chem. Rev. 2001, 101, 4071-4098.

(11) Dyson, H. J.; Wright, P. E.; Scheraga, H. A. The role of hydrophobic interactions in initiation and propagation of protein folding. Proc. Natl. Acad. Sci. U.S.A. 2006, 103, 13057-13061.

(12) Dill, K. A. Dominant forces in protein folding. Biochemistry 1990, 29, 7133-7155.

(13) Tanford, C. The hydrophobic effect and the organization of living matter. Science 1978, 200, 1012-1018.

(14) Kegel, W. K.; van der Schoot, P. Competing hydrophobic and screened-coulomb interactions in Hepatitis B virus capsid assembly. Biophys. J. 2004, 86, 3905-3913.

(15) Kegel, W. K.; van der Schoot, P. Physical regulation of the selfassembly of tobacco mosaic virus coat protein. Biophys. J. 2006, 91, $1501-1512$

(16) Baker, M. B.; Albertazzi, L.; Voets, I. K.; Leenders, C. M. A.; Palmans, A. R. A.; Pavan, G. M.; Meijer, E. W. Consequences of chirality on the dynamics of a water-soluble supramolecular polymer. Nat. Commun. 2015, 6, No. 6234.

(17) Leenders, C. M. A.; Albertazzi, L.; Mes, T.; Koenigs, M. M. E.; Palmans, A. R. A.; Meijer, E. W. Supramolecular polymerization in water harnessing both hydrophobic effects and hydrogen bond formation. Chem. Commun. 2013, 49, 1963-1965.

(18) Leenders, C. M. A.; Baker, M. B.; Pijpers, I. A. B.; Lafleur, R. P. M.; Albertazzi, L.; Palmans, A. R. A.; Meijer, E. W. Supramolecular polymerisation in water; Elucidating the role of hydrophobic and hydrogen-bond interactions. Soft Matter 2016, 12, 2887-2893.

(19) Garzoni, M.; Baker, M. B.; Leenders, C. M. A.; Voets, I. K.; Albertazzi, L.; Palmans, A. R. A.; Meijer, E. W.; Pavan, G. M. Effect of $\mathrm{H}$-bonding on order amplification in the growth of a supramolecular polymer in water. J. Am. Chem. Soc. 2016, 138, 13985-13995. 
(20) Liu, J.; Mendoza, S.; Romàn, E.; Lynn, M. J.; Xu, R.; Kaifer, A. E. Cyclodextrin-modified gold nanospheres. Host-guest interactions at work to control colloidal properties. J. Am. Chem. Soc. 1999, 121, 4304-4305.

(21) Sánchez-Iglesias, A.; Grzelczak, M.; Altantzis, T.; Goris, B.; Pérez-Juste, J.; Bals, S.; Van Tendeloo, G.; Donaldson, S. H., Jr.; Chmelka, B. F.; Israelachvili, J. N.; Liz-Marzán, L. M. Hydrophobic interactions modulate self-assembly of nanoparticles. ACS Nano 2012, 6, 11059-11065.

(22) Grzelczak, M.; Liz-Marzán, L. M. Exploiting hydrophobic interactions at the nanoscale. J. Phys. Chem. Lett. 2014, 5, 2455-2463.

(23) Choueiri, R. M.; Klinkova, A.; Thérien-Aubin, H.; Rubinstein, M.; Kumacheva, E. Structural transitions in nanoparticle assemblies governed by competing nanoscale forces. J. Am. Chem. Soc. 2013, 135, 10262-10265.

(24) Chandler, D. Interfaces and the driving force of hydrophobic assembly. Nature 2005, 437, 640-647.

(25) Arshady, R. Suspension, emulsion, and dispersion polymerization: A methodological survey. Colloid Polym. Sci. 1992, 270, $717-$ 732.

(26) Verwey, E. J. W.; Overbeek, J. T. G. Theory of the Stability of Lyophobic Colloids, 2nd ed.; Dover Publications: Mineola, New York, 1999.

(27) van Ravensteijn, B. G. P.; Kamp, M.; van Blaaderen, A.; Kegel, W. K. General route toward chemically anisotropic colloids. Chem. Mater. 2013, 25, 4348-4353.

(28) Monthéard, J. P.; Jegat, C.; Camps, M. Vinylbenzyl chloride (chloromethylstyrene), polymers, and copolymers. Recent reactions and applications. J. Macromol. Sci., Polym. Rev. 1999, 135-174.

(29) Dormidontova, E. E. Role of competitive PEO-water and water-water hydrogen bonding in aqueous solution PEO behavior. Macromolecules 2002, 35, 987-1001.

(30) Berne, B. J.; Pecora, R. Dynamic Light Scattering: With Applications to Chemistry, Biology, and Physics, 1st ed.; Dover Publications: Mineola, New York, 2000.

(31) van Houtem, M. H. C. J.; Benaskar, F.; Fitié, C. F. C.; MartínRapún, R.; Vekemans, J. A. J. M.; Meijer, E. W. Helical self-assembly and co-assembly of fluorinated, preorganized discotics. Org. Biomol. Chem. 2012, 10, 5898-5908.

(32) Kraft, D. J.; Vlug, W. S.; van Kats, C. M.; van Blaaderen, A.; Imhof, A.; Kegel, W. K. Self-assembly of colloids with liquid protrusions. J. Am. Chem. Soc. 2009, 131, 1182-1186.

(33) Albertazzi, L.; Martinez-Veracoechea, F. J.; Leenders, C. M. A.; Voets, I. K.; Frenkel, D.; Meijer, E. W. Spatiotemporal control and superselectivity in supramolecular polymers using multivalency. Proc. Natl. Acad. Sci. U.S.A. 2013, 110, 12203-12208.

(34) Thomas, J. C. The determination of log normal particle size distributions by dynamic light scattering. J. Colloid Interface Sci. 1987, $117,187-192$.

(35) Wang, J.; Wu, C. Reexamination of the origin of slow relaxation in semi dilute polymer solutions - reptation related or not? Macromolecules 2016, 49, 3184-3191.

(36) Ohshima, H. A simple expression for Henry's function for the retardation effect in electrophoresis of spherical colloidal particles. J. Colloid Interface Sci. 1994, 168, 269-271. 